\title{
SUTURELESS CORONARY ARTERY BYPASS WITH BIOLOGIC GLUED ANASTOMOSES: PRELIMINARY IN VIVO AND IN VITRO RESULTS
}

Steven R. Gundry, MDa

Kirby Black, $\mathrm{PhD}^{\mathrm{b}}$

Hironori Izutani, MD
Objective: As heart surgery becomes increasingly focused on minimally invasive techniques, it has become apparent that conventional techniques of anastomosis will need to be severely altered or abandoned. Toward that end, we developed and tested in vitro and in vivo coronary artery bypass graft anastomoses using a biologic glue formulated from bovine albumin and glutaraldehyde. We used a double-balloon catheter as a temporary internal stent to create and seal the anastomosis during gluing.

Methods: Initially, anastomoses were made between cryopreserved human saphenous vein segments and coronary arteries in vitro on 12 intact bovine hearts. A total of 42 anastomoses were created with the catheter system introduced into the distal end of the graft, exiting the back wall, and entering the anterior wall of the coronary artery. Two balloons (one in the graft and one in the coronary artery) held the anastomosis stable while the biologic glue was applied externally and allowed to set for 2 minutes. The balloon catheter was then removed from the end of the graft simulating a sideto-side internal thoracic artery anastomosis. After the graft had been flushed to assure distal end patency, the open end of the graft was clipped, turning the anastomosis into an end-to-side graft. A pressure transducer was then attached to the graft and saline solution forcefully infused.

Results: All grafts easily held a pressure of $300 \mathrm{~mm} \mathrm{Hg} ; 10$ grafts were tested up to $560 \mathrm{~mm} \mathrm{Hg}$ without leaks. Distal and proximal coronary artery patency was checked by examining flow out of the coronary ostia and by cutting arteries distal to the grafts. All anastomoses were patent on being opened and no glue was seen intraluminally. Subsequently, 3 anastomoses of the left internal thoracic artery to the left anterior descending artery have been constructed in goats, with autopsies at 24 hours, 10 months, and 1 year revealing patent anastomoses.

Conclusion: A biologic glue and catheter system has been developed that allows a coronary anastomosis with a high bursting strength to be performed. When the system has been further developed and tested, truly minimally invasive heart surgery may be possible. (J Thorac Cardiovasc Surg 2000;120:473-7)
From Loma Linda University Medical Center, Loma Linda, Calif, ${ }^{\mathrm{a}}$ and CryoLife, Inc, Marietta, Ga. ${ }^{\mathrm{b}}$

Read at the Seventy-ninth Annual Meeting of The American Association for Thoracic Surgery, New Orleans, La, April 18-21, 1999.

Received for publication April 22, 1999; revisions requested Sept 8, 1999; revisions received May 15, 2000; accepted for publication May 15, 2000.

Address for reprints: Steven R. Gundry, MD, Professor and Head, Division of Cardiothoracic Surgery, Loma Linda University, 11175 Campus St, Loma Linda, CA 92354 (E-mail: sgundry@som.llu.edu).

Copyright (C) 2000 by The American Association for Thoracic Surgery

0022-5223/2000\$12.00+ $0 \quad \mathbf{1 2 / 6 / 1 0 8 5 9 6}$

doi: $10.1067 / \mathrm{mtc} .2000 .108596$
$\Lambda$ s minimally invasive techniques become increasingA ly common in cardiac operations, the limits of the sutured anastomosis in coronary artery bypass grafting are becoming clear. Although the sutured anastomosis on the arrested heart remains the gold standard by which all other techniques are measured, alternative methods of anastomosing the internal thoracic artery (ITA) or saphenous vein to the coronary artery are being attempted. Various stabilizing devices now permit facile anastomoses to be performed on the beating heart and various stapling devices for joining two blood vessels are now in common use elsewhere in the body. ${ }^{1-4}$ Despite these advances, truly minimally invasive coro- 
nary artery bypass must be done through tiny ports without the use of cardiopulmonary bypass and with patency rates that must rival those of current or proposed stent technology. An anastomosis constructed with a biologic or other glue would enable many of these minimally invasive operations to be performed, but to date the use of glues in this fashion has been disappointing. ${ }^{5,6}$ This article describes our use of BioGlue surgical adhesive (CryoLife, Inc, Marietta, Ga) in both in vitro construction of coronary artery anastomoses and the subsequent in vivo application of this technique in experimental animals.

\section{Materials and methods}

BioGlue surgical adhesive is a proprietary compound that combines glutaraldehyde and concentrated bovine albumin with distinct properties that make it useful for consideration as a biologic adhesive for sutureless anastomosis. The compound does not become active until the two agents are mixed within the applicator tip, but once mixed, a set of approximately $65 \%$ of final holding power is achieved within 20 seconds; full holding power is achieved within 2 minutes. This occurs regardless of temperature and occurs under water as well as with air exposure while creating a strong bond. The glue can be removed by "peeling" it off tissues and instruments. Moreover, the compound appears biologically inert, with repaired sheep dissections showing the glue to be present at least 3 months after use. ${ }^{7}$ This agent is approved for use in Europe for the treatment of acute aortic dissection and for facilitating sutured anastomoses of the carotid artery, but it remains under investigation in the United States only for treatment of acute aortic dissection.

To facilitate sutureless coronary artery anastomoses, we procured 12 bovine hearts from the slaughterhouse and rinsed the coronary arteries with a saline-heparin solution. Thawed, previously cryopreserved segments of human saphenous vein (CryoLife) that were unacceptable for human use were used as the conduit material. A total of 42 anastomoses were created with a catheter system that consisted of modified angioplasty balloon catheters (Ideas for Medicine, St Petersburg, Fla) (Fig 1). In brief, the saphenous vein and left coronary artery to be bypassed were brought parallel to each other. Incisions were made on the back of the saphenous vein and the anterior portion of the coronary artery to perform a side-to-side anastomosis. One catheter was introduced into the distal end of the saphenous vein, brought out through the back wall incision of the saphenous vein, and placed into the coronary artery to be bypassed. A second catheter was also introduced into the distal end of the saphenous vein and was placed within the body of the saphenous vein adjacent to the anastomosis to maintain the luminal integrity of the saphenous vein graft. Both balloons were then inflated, so that the two vessels were secured together. The BioGlue adhesive was applied with its standard applicator gun and tip and allowed to set for 2 minutes. After 2 minutes, the balloons were deflated and the catheters removed. The proximal end of the vein graft was fitted with a standard vein graft cannula (Medtronic/DLP, Grand Rapids, Mich) and connected to a pressure-transducing box (Medtronic/DLP). Saline solution was then injected into the proximal vein graft and flow through the distal end of the vein graft assured. Once distal flow through the vein graft was established, the distal end of the graft was clipped with a medium clip (Ligaclip; Ethicon, Inc, Cincinnati, Ohio). The vein graft and coronary arteries were then perfused with a large syringe connected in a $\mathrm{Y}$ fashion to the proximal vein graft and the pressure transducer, and saline solution was forcefully injected to achieve a pressure of at least $300 \mathrm{~mm}$ $\mathrm{Hg}$. Flow was ascertained through the proximal artery by observing saline solution coming from the coronary ostia and from the distal artery by cutting the heart in a bread loaf slice near the apex of the heart and assessing runoff. Saline solution continued to be infused forcefully to as high a pressure as could be achieved competing with the natural runoff through the coronary arteries.

After in vitro testing, a long-term animal model was established. Yearling goats weighing approximately $50 \mathrm{~kg}$ were used as the experimental animal. A small left anterior thoracotomy was performed and the left or right ITA (or both) was harvested by means of a direct-vision technique. After a suitable length had been obtained, the artery was ligated distally and flushed with heparin solution. Through the same anterior limited thoracotomy, the pericardium was opened and purse-string sutures were placed on the ascending aorta and right atrial appendage. Goats were given a 3$\mathrm{mg} / \mathrm{kg}$ dose of sodium heparin and placed on cardiopulmonary bypass. The ascending aorta was crossclamped and a single 500-mL dose of Roe crystalloid cardioplegic solution was administered to produce cardiac arrest. The left anterior descending (LAD) coronary artery was opened in its mid portion. Similarly, as described above, the underside of the ITA was opened approximately $1 \mathrm{~cm}$ from its distal end. The same modified angioplasty balloon catheters were then placed into the distal ITA, one passing through the intended anastomotic site into the LAD, and the other passing up approximately $2 \mathrm{~cm}$ into the ITA adjacent to the anastomotic site (Fig 1). Two 7-0 Prolene stay sutures (Ethicon) were placed laterally on either side of the anastomosis to facilitate orientation of the balloons. Balloons were inflated and BioGlue adhesive was applied around the anastomosis and allowed to set for 2 minutes. Additional BioGlue adhesive was placed on the heart to assess its long-term reactivity. After 2 minutes, the catheter balloons were deflated and the catheters were withdrawn. Flow through the ITA was assured and then a Ligaclip was applied to the distal end of the ITA graft, turning the anastomosis from a side-to-side anastomosis to an end-to-side anastomosis. The crossclamp was removed from the heart. The hearts were defibrillated into a normal sinus rhythm and the animals were weaned uneventfully from cardiopulmonary bypass. Heparin was reversed with protamine sulfate and the chest was closed. An autopsy of 1 animal was done 24 hours after the operation to assess early results; two other animals were allowed to recover and autopsies were done 10 months and 1 year after the procedure. The 


\section{Internal Thoracic a.}

1.

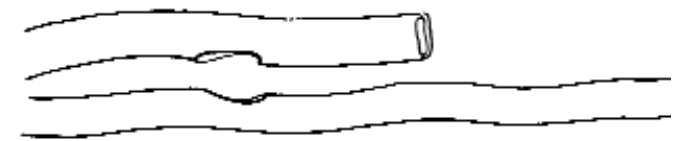

Lt. ant. descending a.

2.

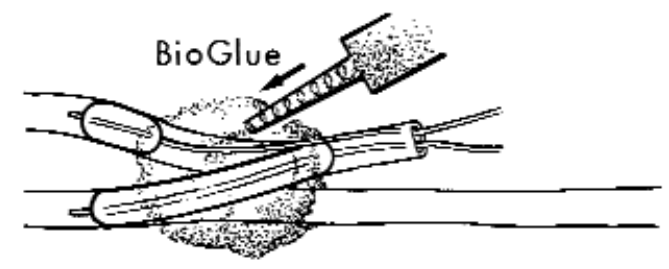

3.

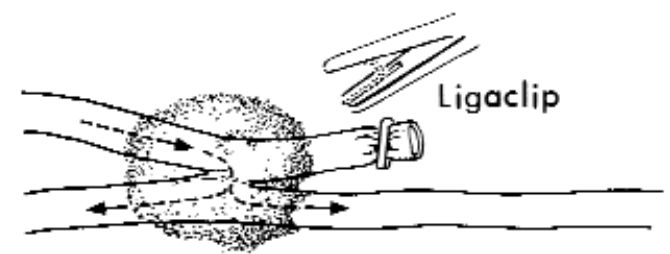

Fig 1. Artist's conception of the anastomosis created with biologic glue. 1, The ITA is placed parallel to the LAD coronary artery. Incisions made on the underside of the ITA and the exposed surface of the LAD. 2, An angioplasty-type balloon catheter is advanced through the arteriotomies and inflated. A second catheter is advanced up the ITA and inflated to preserve luminal integrity. BioGlue adhesive is dispensed over the anastomosis and allowed to set for 2 minutes. 3, Catheters are withdrawn, distal ITA flow is observed, and the tip of the ITA is clipped to convert the side-to-side anastomosis to an end-to-side anastomosis.

animals were fed a normal diet and received humane care in compliance with the "Guide for the Care and Use of Laboratory Animals" prepared by the Institute of Laboratory Animal Resources and published by the National Institutes of Health (NIH publication 86-23, revised 1985). All procedures were approved by the Animal Experimentation Committee of Loma Linda University School of Medicine.

\section{Results}

In vitro results. A total of 42 anastomoses were created with the catheter system and BioGlue adhesive. All anastomoses easily withstood an infusion of saline solution at a pressure of $300 \mathrm{~mm} \mathrm{Hg}$, with the highest pressure recorded at $560 \mathrm{~mm} \mathrm{Hg}$. All anastomoses had patent proximal and distal coronary arteries as evidenced by saline solution leaking out the coronary ostia and from the distal cut coronary artery. No leaks were detected at the site of the BioGlue anastomosis with infusion of saline solution. Macroscopically, no glue was found intraluminally. No histologic sections were performed.

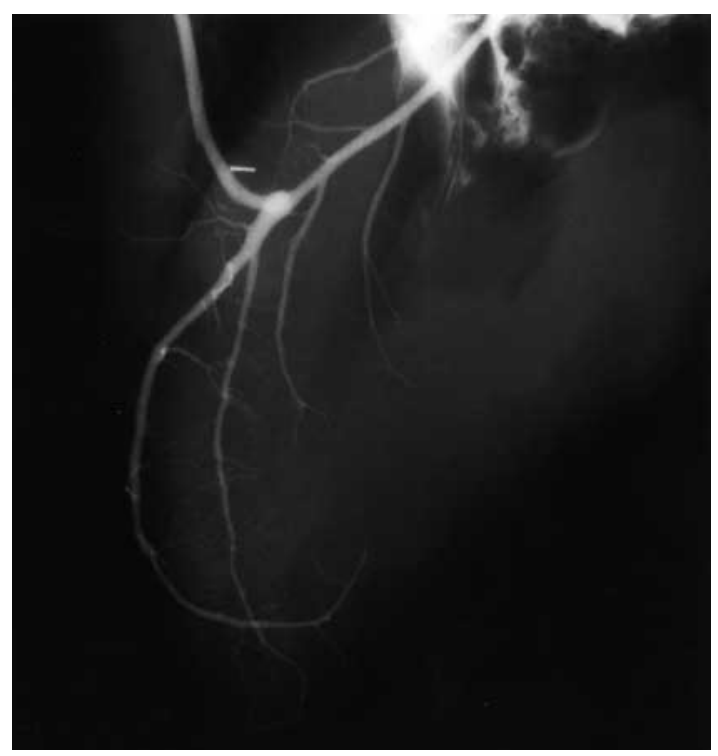

Fig 2. Coronary angiogram of left ITA-LAD coronary artery anastomosis taken 1 year after the operation. The anastomosis was performed with the use of BioGlue adhesive and balloon catheters as described in the text.

In vivo results. All goat hearts were weaned uneventfully from cardiopulmonary bypass. All three hearts had regained normal contractility in the distribution of the distal LAD coronary artery with an open ITA and the crossclamp still in place. In the first goat heart, the proximal LAD was clipped with a Ligaclip. The autopsy of the subsequent two goats found that the Ligaclip had not completely gone over the proximal LAD, resulting in patent LADs in both goats 2 and 3 at 10 months and 1 year after the operation. All goats were fully heparinized before being put to death. After death, the hearts were carefully dissected out, preserving the ITA pedicle. In goat 1 , there was a patent ITA graft to the LAD and minimal surrounding tissue reaction. In goat 2, 10 months postoperatively, there were minimal pericardial adhesions, and portions of the BioGlue adhesive surrounding the anastomosis were still present surrounded with a thin capsule. The anastomotic site was completely patent, as was the proximal and distal LAD coronary artery. The mid right ITA was occluded because of a technical error of kinking at the graft coming over the pericardium. Goat 3 was put to death 1 year after the operation. The left ITA was completely patent, as was the anastomotic site. A coronary angiogram was performed through the ITA, and results are shown in Fig 2. These results demonstrated a completely patent anastomosis with no areas of stenosis, distortion, or aneurysm formation. As in goat 2 , there were typical pericardial adhesions within the pericardium and there were still areas of BioGlue adhe- 


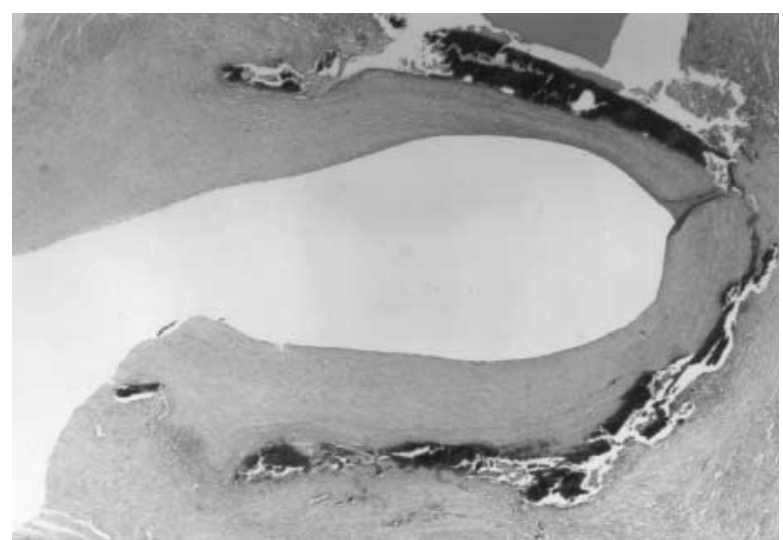

Fig 3. Microscopic section at the junction of the ITA and LAD coronary artery at site of BioGlue application. Note the lack of inflammatory tissue and pieces of intact BioGlue adhesive outside the anastomosis.

sive that had not been absorbed but were merely surrounded by a capsule-like formation. BioGlue adhesive could easily be peeled off the heart where it had been placed 1 year previously. Histologic sections were made, the results of which are shown in Fig 3. No inflammatory reaction to the BioGlue adhesive was found microscopically. The luminal margins were smooth and also without reaction.

\section{Discussion}

These preliminary results demonstrate that a biologic glue made from the combination of bovine albumin and glutaraldehyde is effective in vitro in producing leakfree sutureless coronary artery anastomoses capable of withstanding very high distention pressures, higher certainly than any encountered within human blood pressure ranges. Moreover, our in vivo studies in goats, although small in number, suggest that this anastomosis can be patent 1 full year after construction with BioGlue adhesive. This finding is in contradistinction to earlier attempts with glued or laser-welded anastomoses, which resulted in excessive rates of early thrombosis and early or late suture-line disruption leading to aneurysm formation. Furthermore, this glue is unlike gelatin-resorcin-formol glue, which uses glutaraldehyde, resorcinol, and fibrin to achieve less adhesive qualities. ${ }^{3,5,6}$ If confirmed in a larger series of animals, this sutureless anastomosis created with the aid of a biologic glue opens up tremendous avenues for minimally invasive coronary artery bypass in human beings. The advantages of a glued anastomosis versus a sutured or stapled anastomosis revolve around the potential of delivering the glue through extremely small catheters and using equally small catheters to stabilize the two vessels being anastomosed, as described in this experimental model. With further modification of the vessel-approximating catheters, truly minimally invasive operations based on catheters and biologic glue appear not only possible but feasible.

The lack of a significant inflammatory response and the fact that the goats continued to grow for up to 1 year of follow-up suggest that any detrimental reactivity of the glue to the surrounding tissue is minimal. Although it is unknown whether the same amount of minimal reaction might be found in human beings, the current clinical trials of the glue in aortic dissection have shown no untoward reactions to the glue. Therefore, it is likely that these animal results can be extrapolated to human coronary artery anastomoses quite easily. At the very least, the use of this glue as an adjunct to other forms of rapid anastomosis, whether it be with sutures, stapling, or laser, affords new consideration of these techniques in combination with glue as an alternative to standard anastomoses. ${ }^{1-6}$

\section{Conclusion}

In this brief report, we describe our first experience with BioGlue adhesive (a combination with bovine albumin and glutaraldehyde) as a method of creating a coronary artery anastomosis in both in vitro and in vivo experimental models. Further confirmation of these results would suggest that trials of this glue be undertaken in human beings as a method of achieving truly minimally invasive coronary artery bypass with the potential for long-term patency.

\section{REFERENCES}

1. Calafiore AM, Giammarco GD, Teodori G, et al. Left anterior descending coronary artery grafting via left anterior small thoracotomy without cardiopulmonary bypass, Ann Thorac Surg 1996;61:1658-65.

2. Li Y, Wood MB. End to side anastomosis in the dog using the $3 \mathrm{M}$ precise microvascular anastomotic system: a comparative study. J Reconstr Microsurg 1991;7:345-51.

3. Baser S, Marini CP, Coons MS, et al. Internal mammary coronary artery anastomosis by $\mathrm{CO}_{2}$ laser: an acute experimental study. J Card Surg 1991;6:286-93.

4. Tullekin CA, Verdaasdonk RM, Berendsen W, Mali WPTM. Use of the excimer laser in high flow bypass surgery of the brain. $\mathrm{J}$ Neurosurg 1993;78:477-80.

5. Hoppenstein R, Weissberg D, Goetz RH. Fusiform dilatation and thrombosis of arteries following the application of methyl 2cyanoacrylate (Eastman 910 Monomer). J Neurosurg 1965;23:556-654.

6. Weissberg D, Schwartz P, Goetz RH. Non-suture end to side anastomoses of small blood vessels. Surg Gynecol Obstet 1966;123:341-6.

7. Internal FDA submission data, Cryolife Inc, Kennesaw [GA], 1998. 


\section{Discussion}

Dr Juan C. Chachques (Paris, France). Did you do any histologic studies at the level of the anastomosis? It would be interesting to know what happened to the intima, because some chemical products can induce inflammatory reactions.

Dr Gundry. Those histologic specimens are still under review. I waited a full year to do this last animal's autopsy (which I did last week), so I do not have histologic results. Visually and using loupes we could find no intimal irregularities, and there are no intimal irregularities on the angiogram. However, the histologic information is pending.

Dr James W. Frederiksen (Chicago, Ill). Have you considered using BioGlue surgical adhesive to control unsuturable bleeding, for which fibrin sealant is currently recommended?

Dr Gundry. An investigational device exemption (IDE) trial is underway in the United States for the use of BioGlue adhesive in the treatment of acute aortic dissection. In those cases, the investigators, including me, have used BioGlue adhesive for either the prevention of what we believed would be an uncontrollable leak or the subsequent treatment of a leak uncontrollable by suture. Clinically, it works very well.

Dr Verdi J. DiSesa (Chicago, Ill). You say that this adhesive will stick even in the presence of blood?

Dr Gundry. Yes, it will bind to anything. It will bind to the blood actively, as well as to other tissue. And it will set up under water, so the field does not have to be perfectly dry for this substance to work.

Dr Richard P. Cochran (Madison, Wis). Do you think the double-balloon technique will limit the size of vessel that you can use? As the vessels get smaller and smaller, inserting two balloons may be very difficult.

Dr Gundry. These balloons were actually modified 1- to 2-mm angioplasty balloon catheters. We used them initially in yearling goats weighing about $40 \mathrm{~kg}$, so the ITAs were quite small. At autopsy, these animals weighed approximately $65 \mathrm{~kg}$, so they grew with these anastomoses. This glue does not seem to be detrimental to growth. In fact, the glue was flexible at 1 year.

Dr Alain F. Carpentier (Paris, France). It was not clear from your drawing how you positioned the balloon. Could you elaborate on this technical point?

Dr Gundry. The balloons were both placed through the distal ITA. One balloon was placed in the ITA itself, because earlier trials in vitro had shown that the ITA, if totally decompressed or flattened, would stay in that position after the glue was applied. We merely allowed the ITA to be distended by the balloon to keep it from being compressed.

The second balloon went through the anastomosis and was present within the ITA and the LAD across the anastomosis to seal the anastomosis from glue getting inside and to hold the anastomosis steady. The glue was then applied outside, and it was allowed to set up in 2 minutes. The glue gets about $60 \%$ of its holding power in 20 seconds and about $95 \%$ of its holding power in 2 minutes, so it is a fairly rapid procedure.

Dr Marko I. Turina (Zurich, Switzerland). Other investigators using laser and similar methods found it is simpler to use some stay sutures to support the anastomosis. Did you find this to be the case?

I also have a comment concerning some previous studies that were done, especially in the United Kingdom, with this type of anastomosis, although using laser assistance rather than this method. The fact emerged that early results might be excellent, but there was a very high incidence of late aneurysm at the anastomosis. I would respectfully submit that it is advisable to use a large number of animals and very carefully check for anastomotic aneurysms.

Dr Gundry. I certainly agree with you. We actually used two stay stitches on the outside of the vessels, on the lateral walls, to pull the vessels apart. They were removed after the glue was applied. All the in vitro anastomoses we did totally without sutures. We found it more practical in the living animal to pull the vessel apart with two stay sutures, so those were applied just to hold tissue apart.

Late aneurysm formation is a major problem, particularly with laser-weld anastomoses. That is why I purposely waited so long, 10 months and 1 year, to evaluate these anastomoses for that possibility. Again, the series is extremely small and obviously more experiments in animals have to be done. However, the fact that the glue is still present around the anastomosis at 1 year gives me some encouragement that the late aneurysm formation with other techniques will not be seen in this technique.

Dr DiSesa. Is this is a thin bead of glue or a wad of glue?

Dr Gundry. This is a wad of glue on the outside. It is not purposefully put on the contact area between the two intimal surfaces.

Dr. Mohamed Emara (Cairo, Egypt). How can you exactly fix the size of the ITA and the native coronary artery with your catheter? How can you be sure? And what is the proportion? How much do you open on the ITA and the native artery?

Dr Gundry. We choose a balloon catheter to match the size of the LAD diameter. The balloon catheter actually holds those two vessels apart through that anastomosis and that determines the size of the anastomosis. In other words, if you wanted a 3-mm anastomosis, you would use a 3-mm balloon to spread it that far. If you wanted a 1-mm anastomosis, you would use a 1-mm balloon. It is totally dependent on the balloon size. 